\title{
Long-term outcomes of high dose treatment and autologous stem cell transplantation in follicular and mantle cell lymphomas - a single centre experience
}

\author{
Lucka Boltezar ${ }^{1}$, Karlo Pintaric², Jože Pretnar ${ }^{3}$, Maja Pohar Perme $^{4}$, \\ Barbara Jezersek Novakovic ${ }^{1}$ \\ ${ }^{1}$ Department of Medical Oncology, Institute of Oncology Ljubljana, Slovenia \\ ${ }^{2}$ Faculty of Medicine, University of Ljubljana, Slovenia \\ ${ }^{3}$ Department of Hematology, University Clinical Centre Ljubljana, Slovenia \\ ${ }^{4}$ Department of Biostatistics and Medical Informatics, University of Ljubljana, Slovenia
}

Radiol Oncol 2017; 51(1): 81-87.

Received 6 January 2016

Accepted 24 May 2016

Correspondence to: Assoc. Prof. Barbara Jezeršek Novaković, M.D., Ph.D., Department of Medical Oncology, Institute of Oncology Ljubljana, Zaloška 2, SI-1000 Ljubljana, Slovenia. Phone: +386 15879 631; Fax: +386 15879 305; E-mail: bjezersek@onko-i.si

Disclosure: No potential conflicts of interest were disclosed.

Background. Advanced follicular lymphoma (FL) and mantle cell lymphoma (MCL) are incurable diseases with conventional treatment. The high dose treatment (HDT) with autologous stem cell transplantation (ASCT), however, offers a certain proportion of these patients the prospect of a prolonged disease-free and overall survival. The aim of this study was to investigate the event free survival (EFS) and overall survival (OS) in patients with FL and MCL treated with ASCT.

Patients and methods. Seventeen patients with FL and 29 patients with MCL were included, 15 of them were transplanted to consolidate the response to second line treatment and 24 to consolidate their first remission, respectively. All were conditioned with total body irradiation (TBI) and high dose cyclophosphamide between 2006 and 2014 and all were transplanted with peripheral blood stem cells.

Results. The estimated 5-year OS for FL was 87.8\% (95\% confidence interval [Cl] 59.5\%-96.8\%) and for $\mathrm{MCL} 79.3 \%$ (95\% Cl 56.1\%-91.1\%), respectively. The estimated 5-year EFS for FL was $76.0 \%$ (95\% Cl 48.0\%-90.3\%) and for MCL 69.8\% (95\% Cl 45.5\%-84.8\%), respectively. There were no secondary hematological malignancies observed in either group. Conclusions. Based on above results, the ASCT with TBI is a good treatment option in terms of long-term survival for patients with follicular and mantle cell lymphoma demonstrating a relatively low rate of late toxicities and secondary malignancies.

Key words: follicular lymphoma; mantle cell lymphoma; autologous stem cell transplantation; overall survival; hematological malignancies

\section{Introduction}

Follicular lymphoma (FL) is nowadays still an incurable disease using standard chemotherapy. ${ }^{1}$ Even though it is very sensitive to chemo- and radiotherapy relapses remain the main treatment failure. Significant changes were made in the past two decades, a great gain was the addition of rituximab to the standard CHOP regimen (cyclophosphamide, doxorubicin, vincristine, and prednisone) to prolong progression-free survival (PFS). ${ }^{2,3}$ Rituximab is now the golden standard in the first line treatment ${ }^{2}$, it also improves the overall survival (OS) in relapsed FL patients. ${ }^{4}$ In a Cochrane review in 2012, the authors demonstrated that high dose treatment (HDT) with autologous stem cell 
transplantation (ASCT) improves the progressionfree survival in comparison with chemotherapy or immuno-chemotherapy in previously untreated patients with FL, but does not prolong the OS. ${ }^{5}$ There is also evidence that HDT with ASCT brings benefits to patients with relapsed FL.5,6 A consensus was made in 2013 by the European Group for Blood and Marrow Transplant stating that the SCT is appropriate in patients with first chemo-sensitive relapse to consolidate remission, especially in patients with a short response after immuno-chemotherapy or with high-risk follicular lymphoma international prognostic index (FLIPI). They also pointed out that the HDT with ASCT is appropriate in second or subsequent chemo-sensitive relapses. ${ }^{1}$

Allogeneic transplantation and autologous transplantation are both possible. A higher relapse rate was observed in autologous SCT, whereas no significant difference in the disease-free survival or OS was found. ${ }^{78}$ Allogeneic SCT showed a lower risk for disease recurrence ${ }^{9,10}$, and that also applies for the reduced intensity conditioning SCT. ${ }^{8}$ However, higher treatment-related mortality after allogeneic SCT than after autologous was objectified. One of the factors which contributed significantly to the higher treatment-related mortality was the total body irradiation (TBI). ${ }^{9}$

The mantle cell lymphoma (MCL) is also an incurable disease and successful treatment is still a challenge. In the past few years, many induction regimens were tested for their efficacy. ${ }^{11-14}$ Since 2013, we use in our centre the alternation of rituximab-CHOP (R-CHOP) regimen with R-high dose cytarabine-based regimen for younger patients, as it was shown that it gives a better OS and a higher proportion of partial remission (PR) to complete remission (CR) conversions than other regimens. ${ }^{15}$ Nevertheless, the HDT and ASCT remain an attractive option for those with chemo-sensitive disease regardless of the induction regimen applied. ${ }^{12,14-16}$ In relapsed or refractory disease, long-term disease-free intervals have not been established, the reduced intensity conditioning transplantation is here an option. ${ }^{16}$ Secondary hematological malignancies after the SCT remain an important issue with an estimated 5 year risk of $3.8 \%{ }^{17}$, although some authors report of minimum hematological malignancies or none at all. ${ }^{15}$ The allogeneic SCT offers a lower relapse rate but a higher non-relapse mortality resulting in OS similar to ASCT. ${ }^{18}$ However, it was also shown by Romera et al. that intensive chemotherapy with R-hyper-CVAD (cyclophosphamide, vincristine, doxorubicin, and dexamethasone) alternating with R-high dose methotrexate-cytarabine is also a suitable treatment option for treatment of MCL without ASCT. ${ }^{19}$

The aim of this study was to investigate the event free survival (EFS) and OS in patients with FL and MCL treated with ASCT.

\section{Patients and methods}

The study population includes 17 patients with FL and 29 patients with MCL who underwent ASCT after HDT conditioning with TBI (fractionated $6 \mathrm{x}$ 200 cGy during three days' period) and high dose cyclophosphamide $(2 \times 60 \mathrm{mg} / \mathrm{kg})$ between 2006 and 2014 and whose conventional treatment and the TBI were performed at the Institute of Oncology Ljubljana and the transplantation procedure at the University Clinical Centre Ljubljana, Department of Hematology. The study was conducted according to the Helsinki Declaration, all patients were aged 18 or above and patient's informed consent was obtained.

According to national guidelines, FL patients were transplanted in their second remission and only three patients with adverse prognostic factors were consolidated for their first remission. Conversely, MCL patients were transplanted already in their first remission and exceptionally only five patients (with low risk international prognostic index for MCL [MIPI]) were consolidated for their second or later remission. All patients included in the study were transplanted with stem cells collected from peripheral blood. All patients were also treated with maintenance rituximab post ASCT concordant with the latest studies ${ }^{20,21}$ and were treated with rituximab containing regimens in their induction treatments. Twenty-six MCL patients received $\mathrm{R}-\mathrm{CHOP}$ regimen as their first induction treatment and 3 patients received $\mathrm{R}-\mathrm{CHOP}$ as the second line treatment. In the FL group, only 10 patients started with R-CHOP as the first line induction treatment and 7 patients received $\mathrm{R}-\mathrm{CHOP}$ after failure of first line treatment without rituximab.

The disease status prior to ASCT was evaluated according to revised Cheson's criteria. ${ }^{22}$ The OS was measured from the time of HDT/ASCT to death from any cause. The EFS was measured from the time of HDT/ASCT to progression, recurrence of the disease, or death from any cause. The nonrelapse mortality was defined as death from any cause without progression or relapse of lymphoma. The EFS and OS were calculated according to the Kaplan-Meier method and differences between 
TABLE 1. Patients' characteristics

\begin{tabular}{|c|c|c|}
\hline & $\begin{array}{l}\text { Follicular lymphoma group } \\
\qquad \mathrm{N}=17\end{array}$ & $\begin{array}{l}\text { Mantle cell lymphoma group } \\
\qquad \mathrm{N}=29\end{array}$ \\
\hline $\operatorname{Sex}(F / M)$ & $7(41.2 \%) / 10$ (58.8\%) & 6 (20.7\%) / 23 (79. 3\%) \\
\hline $\begin{array}{l}\text { FL grade } \\
\text { I/II } \\
\text { IIla }\end{array}$ & $\begin{array}{l}13(76.5 \%) \\
4(23.5 \%)\end{array}$ & I \\
\hline Pleomorphic/blastoid MCL & l & $10(34.5 \%)$ \\
\hline $\begin{array}{l}\text { HDT/ASCT } \\
\text { After first line treatment* } \\
\text { After second line treatment or later* }\end{array}$ & $\begin{array}{l}3(17.6 \%) \\
14(82.4 \%)\end{array}$ & $\begin{array}{l}24(82.7 \%) \\
5(17.2 \%)\end{array}$ \\
\hline $\begin{array}{l}\text { Disease status prior HDT/ASCT } \\
\text { CR } \\
\text { PR }\end{array}$ & $\begin{array}{l}5(29.4 \%) \\
12(70.6 \%)\end{array}$ & $\begin{array}{l}13(44.8 \%) \\
16(55.2 \%)\end{array}$ \\
\hline
\end{tabular}

* according to national guidelines; $\mathrm{CR}=$ complete remission; $\mathrm{F}=$ female; $\mathrm{FL}=$ follicular lymphoma; FLIPI = follicular lymphoma international prognostic index; $\mathrm{HDT} / \mathrm{ASCT}=$ high dose treatment/autologous stem cell transplantation; $\mathrm{M}=$ male; $\mathrm{MCL}=$ mantle cell lymphoma; $\mathrm{MIPI}$ = international prognostic index for mantle cell lymphoma; $\mathrm{N}=$ number; $\mathrm{PR}$ = partial remission

subgroups were analyzed by the log-rank test and by the Cox proportional hazards model. The nonrelapse mortality was estimated using the cumulative incidence function. The program used was $R$ Statistical software ( $R$ for Windows, version R-3.1.2, University of Auckland) and GraphPad Prism programme (version 3.02, GraphPad Software, USA).

The survival outcomes of three patients with histologically confirmed transformation of FL into the diffuse large B-cell lymphoma at the time of HDT/ASCT were analyzed separately.

\section{Results}

\section{Patients' characteristics and disease status prior to ASCT}

Patients' characteristics are shown in Table 1. In the FL group, there was a slight male predominance. Median age at diagnosis of lymphoma was 46 years. Thirteen patients had FL grade I/II and 4 patients had FL grade IIIa. None of the patients had FL grade IIIb. Four of them were considered as low risk patients ( $0-1$ points) according to FLIPI, 9 were in the intermediate group ( 2 points) and 4 of them in the high risk group (3-5 points). Three patients $(17.6 \%)$ were transplanted to consolidate their first remission, $14(82.4 \%)$ were transplanted as a consolidation after treatment for their first relapse. Five (29.4\%) of them were in CR before transplantation, 12 (70.6\%) of them had PR. In the MCL group, there were 23 males and 6 females. Median age at diagnosis was 54 years. There were 10 pleomorphic/blastoid variants of MCL in the study population. Twenty-four patients had MIPI below 5.7 (low risk group), 4 patients between 5.7 and 6.2 (intermediate risk group) and one patient was in the high risk group (MIPI above 6.2). Twenty-four patients $(82.7 \%)$ were transplanted in their first remission, 3 patients $(10.3 \%)$ were transplanted to consolidate their second remission and 2 patients $(6.9 \%)$ for later than second remission. Thirteen (44.8\%) of them had CR before transplantation and 16 (55.2\%) of them had PR.

\section{Overall and event free survival}

The estimated 5-year OS for FL was $87.8 \%$ (95\% confidence interval [CI] 59.5\%-96.8\%) and the median OS has not been reached yet with a median follow-up time of 57.5 months (range 15-102 months) (Figure 1A). The FLIPI score did not influence the OS ( $p=0.365$, data not shown) and also the disease status had no significant impact on the OS ( $p=0.570$, hazard ratio (HR) $2.188,95 \%$ CI HR $0.120-47.29)$.

The estimated 5-year OS for MCL was 79.3\% (95\% CI 56.1\%-91.1\%), and the median OS has not been reached yet with a median follow-up time of 31.5 months (range 2.5 months-95.5 months) (Figure 1B). The MIPI index had no impact on OS $(p=0.776$, data not shown) and the same is true for 
Overall surviwal FL

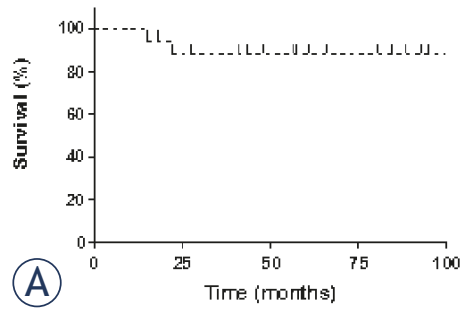

Owerall survival MCL

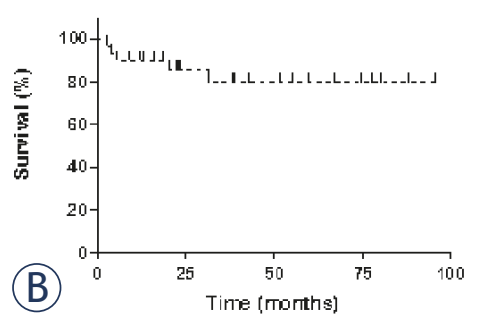

FIGURE 1. (A) Overall survival of follicular lymphoma (FL) patients ( $n=17$ ); (B) Overall survival of mantle cell lymphoma $(M C L)$ patients $(n=29)$.
Event-free surviwalFL

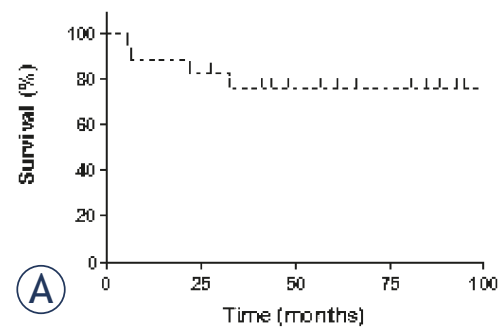

Event-free survival MCL

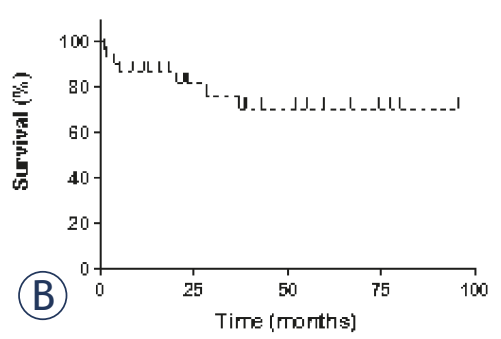

FIGURE 2. (A) Event-free survival of follicular lymphoma (FL) patients ( $n=17)$; (B) event-free survival of mantle cell lymphoma $(M C L)$ patients $(n=29$ ).

the CR or PR status prior to transplantation ( $\mathrm{p}=$ 0.668, HR 0.679, 95\% CI HR 0.117-3.952).

The estimated 5-year EFS for FL was $76.0 \%(95 \%$ CI $48.0 \%-90.3 \%$ ) and is shown in Figure 2A. The FLIPI score did not influence the EFS ( $p=0.124$, data not shown) and also the disease status (CR or PR) prior to ASCT did not ( $\mathrm{p}=0.851$, HR 0.806, 95\% CI HR 0.0936-7.056).

The estimated 5-year EFS for MCL was 69.8\% (95\% CI $45.5 \%-84.8 \%)$ and is shown in Figure 2B. The MIPI index had no impact on the EFS ( $p=$ 0.486, data not shown) and the same holds true for the CR or PR status prior to transplantation ( $\mathrm{p}=$ 0.391, HR 0.485, 95\% CI HR 0.099-2.472).

\section{Non-relapse mortality and secondary malignancies}

In FL group, 2 patients $(11.8 \%)$ died. One patient transformed into the diffuse large-B cell lymphoma and died due to complications of a massive transformation and in one patient the cause of death was unrelated to lymphoma. In MCL group, 5 patients $(17.2 \%)$ died. Two died of progression of MCL, one died due to histologically confirmed JC virus leukoencephalopathy and two of causes unrelated to lymphoma. The estimated probability of non-relapse mortality after 5 years equals 0.063 in FL (95\% CI 0-0.186) and 0.116 in MCL (95\% CI $0-0.245)$.

In the FL group, 1 patient (5.9\%) developed ovarian cancer (treated with surgery) and 1 patient $(5.9 \%)$ bladder cancer, but there were no hematological malignancies. In MCL group, there were also no hematological malignancies, 1 patient was just suspected to have myelodysplastic syndrome due to prolonged thrombocytopenia but this suspicion was later on rejected. One patient $(3.4 \%)$ developed prostate cancer and is receiving hormone treatment.

\section{Transformations}

Survival analyses were done also for the 3 patients with transformation of FL into the diffuse largeB cell lymphoma at the time of HDT and ASCT. Median age of these patients was 42 years (range 39-52). There were 2 males and one female. The FLIPI scores were 0, 1 (low risk group) and 2 (intermediate risk group). The disease status prior transplantation was CR in all three cases, one patient was transplanted to consolidate the second remission and the other two to consolidate their third remission. They were all conditioned with TBI and high dose cyclophosphamide. Two of them died, one is still alive. With a median survival of 39.5 months the difference between the OS of those 3 patients and our FL group was not significant ( $p$ $=0.062$, data not shown). The EFS, however, was in these patients significantly shorter (median EFS of 15 months) than in our FL group ( $p=0.003, \mathrm{HR}$ 6.805, 95\% CI HR 3.414-495.6).

\section{Discussion}

\section{Follicular lymphoma}

The estimated 5-year OS for our FL patients is $87.8 \%$ and the median OS has not been reached. Compared to the study of El-Najjar et al., the survival of our patients is superior since their 5-year OS for TBI-based HDT was 77\% while for carmustine, etoposide, cytarabine, melphalan (BEAM) treated patients it was $74 \%$. The difference between their treatment arms was statistically significant ( $p$ $=0.042) .{ }^{23}$ Due to our relatively small sample our CI is relatively wide, but the results are encouraging.

Our projected 8-year OS was also $87.7 \%$, since we had no further events in that period, but the 8-year estimation is not so reliable as the sample gets smaller. Thereby, our results resemble more than ASCT studies the allogeneic transplantation 
studies, for example a study of Khouri et al, where they reported the 11-year OS of $78 \%$ achieved by the addition of ${ }^{90} \mathrm{Y}$ to the fludarabine and cyclophosphamide conditioning regimen. ${ }^{24}$ Van Besien et al. conducted a study comparing allogeneic and autologous SCT. The TBI conditioning regimen was more used for allogeneic transplantation, which differs from our study. Their 5-year adjusted probabilities of survival after allogeneic transplantation were $51 \%$ and TBI was associated with higher transplant related mortality but a lower recurrence of the disease. ${ }^{9}$ Our study confirms that the TBI including regimen is also appropriate for ASCT with a low rate of secondary malignancies and late toxicities.

The estimated 5-year EFS of our patients was $76.0 \%$ (95\% CI $48.0 \%-90.3 \%$ ) which is also better than in El-Najjar et al. study, where the 5-year EFS for TBI-based HDT was 58\%. Like in case of OS, the EFS was also significantly worse in patients with BEAM-based HDT $\left(49 \%\right.$, p < 0.001). ${ }^{23}$ Eightytwo point four percent of our patients were transplanted for their first relapse, with the HDT and ASCT being an established treatment regimen for relapsed disease in the rituximab era. ${ }^{25-27}$ Sebban $e t$ al. namely showed no benefit of HDT and ASCT after first line treatment regarding the EFS and OS in comparison to standard chemotherapy. ${ }^{25}$

The significant difference in EFS between our patients with FL and those whose lymphomas transformed to the diffuse large-B cell lymphoma $(p=0.0034)$ is interesting despite the fact that our study groups were relatively small. These results emphasize the importance of appropriate patient selection for transplantation procedure as the outcome of the patients with transformation cannot compare to our FL group (at least partially on account of a small number of transformations but also on account of a significantly worse prognosis of patients having the transformation), showing that FL patients with a transformation to the diffuse large-B cell lymphoma are not ideal candidates for TBI and high dose cyclophosphamide followed by ASCT. However, the transformation cannot be predicted and for these patients an alternative salvage option should be sought - for example a different conditioning regimen.

\section{Mantle cell lymphoma}

In our study population, the estimated 5-year OS for MCL was $79.3 \%$ (95\% CI 56.1\%-91.1\%) and the median OS has not been reached. Also in this population, our results are comparable to the ones reported by other authors ${ }^{11,28,29}$, for example by Dreyling et al. where they compared survival after TBI and ASCT versus maintenance with $\alpha$-interferon after completion of induction therapy. The 3-year OS was 83\% after ASCT versus 77\% in the IFN group $(p=0.18) .{ }^{11}$ The prospective trial of Nordic Lymphoma Group, however, reports of a 4 -year OS of $81 \%$ and a 4 -year EFS of $63 \%$, but their patients were conditioned with the BEAM regimen. ${ }^{30}$ We can also estimate the 7 -year OS for our MCL as $79.3 \%$ since there were no additional events, but that estimation is not as reliable as the 5-year OS.

In a study by Oinonen et al., the median EFS was set at 39 months ${ }^{31}$, while in our group the median has not been achieved yet with a median observation period of 31.5 months. Delarue et al. revealed the same, comparing the BEAM treatment to TBI the median EFS of patients receiving BEAM was 55 months, whereas the median EFS for the TBI regimen group has not been reached $(p=0.05) .{ }^{15}$ This again proves that TBI conditioning regimen is safe and appropriate for ASCT in MCL.

The ASCT performed in line of first line treatment to consolidate the first remission was documented to have a significantly better EFS and OS compared to delayed ASCT. ${ }^{11,32}$ In our study, the impact of early or late transplantation on OS and EFS was not statistically significant (OS: $p=0.141$, HR 0.284, 95\% CI HR 0.015-1.82; EFS: $\mathrm{p}=0.393$, HR 0.497 , 95\% CI HR 0.057-3.080). That could be due to our relatively small study group and a small proportion of transplantations to consolidate the second or further remissions. Most of our patients were namely transplanted after first line treatment.

\section{TBI conditioning}

The TBI regimen has been linked to secondary malignancies, mostly hematological - myelodysplastic syndromes (MDS) and acute myeloid leukemias (AML). ${ }^{17,26,33-35}$ The numbers vary among different studies - they range from a 5-year estimated risk of $3.8 \%{ }^{17}$ to a 7 -year cumulative probability of $8.9 \%{ }^{34}$ and a 6 -year incidence of $12 \% .^{33}$ The therapy-related-MDS/therapy-related-AML (t-MDS/t-AML) also depended on amount of radiation used for TBI. In Metayer et al. multicenter case-control study, there was a nonsignificant excess risk of tMDS/ tAML with use of conditioning regimens with TBI, compared with no TBI (relative risk [RR] = 2.0; 95\% CI 0.95-4.18; $\mathrm{p}=0.07)$. The dose-response analysis, however, revealed that the excess risk was limited to patients receiving TBI doses of 13.2 
Gy $(R R=6.6 ; p=0.003)$. Those with lower TBI doses (5-12 Gy) had no evidence of elevated risk of tMDS/tAML. ${ }^{34}$ Hypotheses on cellular level are that HDT and TBI cause similar cellular and genetic damages as the pre-transplant therapy to the hematopoietic progenitor cells and therefore provide opportunity for causing damage to the stem cells. Reinfusion of peripheral blood progenitor cells with previously damaged DNA can result in clonal abnormal hematopoiesis. ${ }^{35}$ In this study, we had no hematological malignancies in either of our groups. Even though the number of our patients is relatively small we may assume that TBI with high doses of cyclophosphamide is a safe method of conditioning prior to transplantation in regard of the secondary hematological malignancies.

\section{Conclusions}

Follicular lymphoma and mantle cell lymphoma remain incurable diseases even in modern era. Treatment modalities are improving and transplantation of stem cells is gaining a more and more important role each year. We present good long term survivals after autologous stem cell transplantation following conditioning with TBI and high dose cyclophosphamide with minimal toxicities and low incidence of secondary malignancies. Our study also demonstrates a low relapse rate and therefore we conclude that the autologous stem cell transplantation represents a reliable option for treatment of follicular and mantle cell lymphoma with good long term results.

\section{Acknowledgments}

This study was partially financed by Ministry of Science and Technology of Slovenia - grant P30321.

\section{References}

1. Montoto S, Corradini P, Dreyling M, Ghielmini M, Kimby E, López-Guillermo A, et al. Indications for hematopoietic stem cell transplantation in patients with follicular lymphoma: a consensus project of the EBMT-Lymphoma Working Party. Hematologica 2013; 98: 1014-21.

2. Fisher RI, LeBlanc M, Press OW, Maloney DG, Unger JM, Miller TP. New treatment options have changed the survival of patients with follicular lymphoma. J Clin Oncol 2005; 23: 8447-52.

3. Bachy E, Houot R, Morschhauser F, Sonet A, Brice P, Belhadj K, et al. Longterm follow up of the FL2000 study comparing CHVP-interferon to CHVP interferon plus rituximab infollicular lymphoma. Hematologica 2013; 98 : 1107-14.
4. Vidal L, Gafter-Gvili A, Salles G, Dreyling MH, Ghielmini M, Hsu Schmitz SF, et al. Rituximab maintenance for the treatment of patients with follicular lymphoma: an updated systematic review and meta-analysis of randomized trials. J Nat/ Cancer Inst 2011; 103: 1799-806.

5. Schaaf M, Reiser M, Borchmann P, Engert A, Skoetz N. High-dose therapy with autologous stem cell transplantation versus chemotherapy or immuno-chemotherapy for follicular lymphoma in adults. Cochrane Database Syst Rev 2012; 1: CD007678 doi: 10.1002/14651858.

6. Schouten HC, Qian W, Kvaloy S, Porcellini A, Hagberg H, Johnsen HE, et al. High-dose therapy improves progression-free survival and survival in relapsed follicular non-Hodgkin's lymphoma: results from the randomized European CUP trial. J Clin Oncol 2003; 21: 3918-27.

7. Ingram W, Devereux S, Das-Gupta EP, Russell NH, Haynes AP, Byrne JL, et al. Outcome of BEAM-autologous and BEAM-alemtuzumab allogeneic transplantation in relapsed advanced stage follicular lymphoma. Br J Haematol 2008; 141: 235-43.

8. Robinson SP, Canals $\mathrm{C}$, Luang J, Tilly $\mathrm{H}$, Crawley $\mathrm{C}$, Cahn JY, et al. The outcome of reduced intensity allogeneic stem cell transplantation and autologous stem cell transplantation when performed as a first transplant strategy in relapsed follicular lymphoma: an analysis from the Lymphoma Working Party of the EBMT. Bone Marrow Transplant 2013; 48: 1409-14.

9. van Besien K, Loberiza FR Jr, Bajorunaite R, Armitage JO, Bashey A, Burns $\sqcup$, et al. Comparison of autologous and allogeneic hematopoietic stem cell transplantation for follicular lymphoma. Blood 2003; 102: 3521-9.

10. Tomblyn MR, Ewell M, Bredeson C, Kahl BS, Goodman SA, Horowitz MM, et al. Autologous versus reduced-intensity allogeneic hematopoietic cell transplantation for patients with chemosensitive follicular non-Hodgkin lymphoma beyond first complete response or first partial response. Biol Blood Marrow Transplant 2011; 17: 1051-7.

11. Dreyling M, Lenz G, Hoster E, Van Hoof A, Gisselbrecht C, Schmits R, et al. Early consolidation by myeloablative radiochemotherapy followed by autologous stem cell transplantation in first remission significantly prolongs progression-free survival in mantle-cell lymphoma: results of a prospective randomized trial of the European MCL Network. Blood 2005; 105: 2677-84.

12. Witzig TE. Current treatment approaches for mantle-cell lymphoma. J Clin Oncol 2005; 23: 6409-14.

13. Merli F, Luminari S, llariucci F, Petrini M, Visco C, Ambrosetti A, et al. Rituximab plus HyperCVAD alternating with high dose cytarabine and methotrexate for the initial treatment of patients with mantle cell lymphoma, a multicentre trial from Gruppo Italiano Studio Linfomi. Br J Haematol 2012; 156: 346-53.

14. lams W, Reddy MN. Consolidative autologous hematopoietic stem-cell transplantation in first remission for non-Hodgkin lymphoma: current indications and future perspective. Ther Adv Hematol 2014; 5: 153-67.

15. Delarue R, Haioun C, Ribrag V, Brice P, Delmer A, Tilly H, et al. CHOP and DHAP plus rituximab followed by autologous stem cell transplantation in mantle cell lymphoma: a phase 2 study from the Groupe d'Etude des Lymphomes de l'Adulte. Blood 2013; 121: 48-53.

16. Lunning MA, Armitage JO. The place of transplantation in mantle cell lymphoma. Oncology 2013; 27: 2-6.

17. Lenz G, Dreyling M, Schiegnitz E, Haferlach T, Hasford J, Unterhalt M, et al. Moderate increase of secondary hematologic malignancies following myeloablative radiochemotherapy and autologous stem cell transplantation in patients with indolent lymphoma: results of a prospective randomized trial of the German Low Grade Lymphoma Study Group (GLSG). J Clin Oncol 2004; 22: 4926-33.

18. Bhatt VR, Vose JM. Hematopoietic stem cell transplantation for non-Hodgkin lymphoma. Hematol Oncol Clin North Am 2014; 28: 1073-95.

19. Romaguera JE, Fayad LE, Feng L, Hartig K, Weaver P, Rodriguez MA, et al. Ten-year follow-up after intense chemoimmunotherapy with RituximabHyperCVAD alternating with Rituximab-high dose methotrexate/cytarabine (R-MA) and without stem cell transplantation in patients with untreated aggressive mantle cell lymphoma. Br J Haematol 2010; 150: 200-8.

20. Dreyling M, Geisler C, Hermine O, Kluin-Nelemans HC, Le Gouill S, Rule S, et al. ESMO Guidelines Working Group. Newly diagnosed and relapsed mantle cell lymphoma: ESMO Clinical Practice Guidelines for diagnosis, treatment and follow-up. Ann Oncol 2014; 25: 83-92. 
21. Pettengell R, Schmitz N, Gisselbrecht C, Smith G, Patton WN, Metzner $B$, et al. Rituximab purging and/or maintenance in patients undergoing autologous transplantation for relapsed follicular lymphoma: a prospective randomized trial from the Lymphoma Working Party of the European Group for Blood and Marrow Transplantation. J Clin Oncol 2013; 31: 1624-30.

22. Cheson BD, Pfistner D, Juweid ME, Gascoyne RD, Specht L, Horning SJ, et al. Revised response criteria for malignant lymphoma. J Clin Oncol 2007; 25: $579-86$

23. El-Najjar I, Boumendil A, Luan JJ, Bouabdallah R, Thomson K, Mohty M, et al. The impact of total body irradiation on the outcome of patients with follicular lymphoma treated with autologous stem-cell transplantation in the modern era: a retrospective study of the EBMT Lymphoma Working Party. Ann Oncol 2014; 25: 2224-9.

24. Khouri IF, Saliba RM, Erwin WD, Samuels BI, Korbling M, Medeiros $\sqcup$, et al. Nonmyeloablative allogeneic transplantation with or without ${ }^{90} \mathrm{yttrium}$ ibritumomab tiuxetan is potentially curative for relapsed follicular lymphoma: 12-year results. Blood 2012; 119: 6373-8.

25. Sebban C, Mounier N, Brousse N, Belanger C, Brice P, Haioun C, et al. Standard chemotherapy with interferon compared with CHOP followed by high-dose therapy with autologous stem cell transplantation in untreated patients with advanced follicular lymphoma: the GELF-94 randomized study from the Groupe d'Etude des Lymphomes de l'Adulte (GELA). Blood 2006; 108: $2540-4$.

26. Rohatiner AZ, Nadler L, Davies AJ, Apostolidis J, Neuberg D, Matthews J, et al. Myeloablative therapy with autologous bone marrow transplantation for follicular lymphoma at the time of second or subsequent remission: longterm follow-up. J Clin Oncol 2007; 25: 2554-9.

27. Le Gouill S, De Guibert S, Planche L, Brice P, Dupuis J, Cartron G, et al. Impact of the use of autologous stem cell transplantation at first relapse both in naïve and previously rituximab exposed follicular lymphoma patients treated in the GELA/GOELAMS FL2000 study. Hematologica 2011; 96: 1128-35.

28. Damon LE, Johnson JL, Niedzwiecki D, Cheson BD, Hurd DD, Bartlett NL, et al. Immunochemotherapy and autologous stem-cell transplantation for untreated patients with mantle-cell lymphoma: CALGB 59909. J Clin Oncol 2009; 27: 6101-8.

29. Szcześniak M, Armatys A, Kurzawa R, Kandzia T, Kozioł D, Frankiewicz A, et al. Autologous stem cell transplantation for mantle cell lymphoma - single centre experience. Contemp Oncol (Pozn) 2013; 17: 456-9.

30. Geisler CH, Kolstad A, Laurell A, Andersen NS, Pedersen LB, Jerkeman M, et al. Long-term progression-free survival of mantle cell lymphoma after intensive front-line immunochemotherapy with in vivo-purged stem cell rescue a nonrandomized phase 2 multicenter study by the Nordic Lymphoma Group. Blood 2008; 112: 2687-93.

31. Oinonen $R$, Jantunen $E$, Itälä $M$, Lehtinen $T$, Kuittinen $O$, Franssila $K$, et al. Autologous stem cell transplantation in patients with mantle cell lymphoma. Leuk Lymphoma 2002; 43: 1229-37.

32. Dreger P, Martin S, Kuse R, Sonnen R, Glass B, Kröger N, et al. The impact of autologous stem cell transplantation on the prognosis of mantle cell lymphoma: a joint analysis of two prospective studies with 46 patients. Hematol J 2000; 1: 87-94.

33. Micallef IN, Lillington DM, Apostolidis J, Amess JA, Neat M, Matthews J, et al. Therapy-related myelodysplasia and secondary acute myelogenous leukemia after high-dose therapy with autologous hematopoietic progenitor-cell support for lymphoid malignancies. J Clin Oncol 2000; 18: 947-55.

34. Metayer C, Curtis RE, Vose J, Sobocinski KA, Horowitz MM, Bhatia S, et al. Myelodysplastic syndrome and acute myeloid leukemia after autotransplantation for lymphoma: a multicentre case-control study. Blood 2003; 101: 2015-23.

35. Akhtari M, Bhatt VR, Tandra PK, Krishnamurthy J, Horstman H, Dreessen A et al. Therapy-related myeloid neoplasms after autologous hematopoietic stem cell transplantation in lymphoma patients. Cancer Biol Ther 2013; 14: 1077-88. 